\title{
Low-loss design for the high-intensity accumulator ring of the Spallation Neutron Source
}

\author{
J. Wei, D. T. Abell, J. Beebe-Wang, M. Blaskiewicz, P. R. Cameron, N. Catalan-Lasheras, G. Danby, A. V. Fedotov, \\ C. Gardner, J. Jackson, Y. Y. Lee, H. Ludewig, N. Malitsky, W. Meng, Y. Papaphilippou, D. Raparia, N. Tsoupas, \\ W. T. Weng, R. L. Witkover, and S. Y. Zhang \\ Brookhaven National Laboratory, Upton, New York 11973 \\ (Received 29 January 2000; published 31 August 2000)
}

\begin{abstract}
This paper summarizes the low-loss design for the Spallation Neutron Source accumulator ring ["Spallation Neutron Source Design Manual" (unpublished)]. A hybrid lattice consisting of FODO arcs and doublet straights provides optimum matching and flexibility for injection and collimation. For this lattice, optimization focuses on six design goals: a space-charge tune shift low enough (below 0.15) to avoid strong resonances, adequate transverse and momentum acceptance for efficient beam collimation, injection optimized for desired target beam shape and minimal halo development, compensation of magnet field errors, control of impedance and instability, and prevention against accidental system malfunction. With an expected collimation efficiency of more than $90 \%$, the uncontrolled fractional beam loss is expected to be at the $10^{-4}$ level.
\end{abstract}

PACS numbers: 29.27.Bd, 41.85.-p, 29.27.Ac

\section{INTRODUCTION}

In recent years, high-intensity ion beams have been proposed for a wide variety of applications. These include spallation neutron sources, neutrino factories, transmutation of nuclear waste, heavy ion fusion, and muon collider drivers [1]. Beam power in these machines, usually $1 \mathrm{MW}$ or more, is 1 order of magnitude above that of existing accelerator facilities. In the design of such next-generation facilities, the primary concern is that radioactivation caused by excessive uncontrolled beam loss can limit a machine's availability and maintainability. Based on operational experience at the LAMPF linac [2] at Los Alamos National Laboratory and the alternating gradient synchrotron (AGS) and Booster [3] at Brookhaven National Laboratory, hands-on maintenance [4] demands an average uncontrolled beam loss not exceeding a couple of watts of beam power per tunnel meter. At megawatt power levels, this corresponds to a fractional beam loss of $10^{-6}$ per meter. Equivalently, for a storage ring several hundred meters in circumference, the tolerable fractional beam loss is about $10^{-4}$.

Existing proton synchrotrons and accumulator rings have beam losses as high as several tens of percent, mostly at injection, capture, and initial ramping. The smallest beam loss is about $3 \times 10^{-3}$, achieved at the proton storage ring (PSR) at the Los Alamos National Laboratory. This loss value, however, is still more than 1 order of magnitude higher than desired for next-generation machines.

Uncontrolled beam losses are usually attributed to (1) a high space charge tune shift ( 0.25 or larger) at injection, (2) limited physical and momentum acceptance, (3) premature $\mathrm{H}^{-}$and $\mathrm{H}^{0}$ stripping and injection foil scattering, (4) large magnet field errors, misalignments, and dipole-quadrupole matching errors [for rapid cycling syn- chrotrons (RCS)], (5) instabilities (e.g., PSR instability), and (6) accidental injection and extraction malfunction. A low-loss design must carefully address these six issues.

The Spallation Neutron Source (SNS) is based on an accelerator producing an average proton beam power of $2 \mathrm{MW}$ at a repetition rate of $60 \mathrm{~Hz}[5,6]$. Table I compares the main parameters of the SNS and some other existing and proposed neutron facilities. Table II lists the main parameters of the SNS ring. During 1999, the first year of construction, a study was performed comparing a full-energy linac with accumulator ring to an RCS. As opposed to an RCS, an accumulator ring simplifies the capture process and avoids ramping complications. The study concluded that, especially due to the stringent beam-loss limit of a 2 MW source, the required RCS design is technically demanding and consequently less cost effective [7]. The SNS accelerator complex now comprises a source and front end, a $1 \mathrm{GeV}$ full-energy linac, an accumulator ring, and its transport lines. With a circumference of $220 \mathrm{~m}$, the accumulator ring compresses the proton beam into $0.6 \mu \mathrm{s}$ pulses of $2 \times 10^{14}$ particles, and delivers them at a rate of $60 \mathrm{~Hz}$ to a liquid mercury target for spallation neutron production.

TABLE I. Main parameters of some existing and proposed accelerator-based neutron facilities.

\begin{tabular}{llccc}
\hline \hline \multicolumn{1}{c}{ Machine } & $\begin{array}{c}\text { Energy } \\
(\mathrm{GeV})\end{array}$ & $\begin{array}{c}\text { Intensity } \\
(\mathrm{ppp})\end{array}$ & $\begin{array}{c}\text { Rep rate } \\
(\mathrm{Hz})\end{array}$ & $\begin{array}{c}\text { Power } \\
(\mathrm{MW})\end{array}$ \\
\hline \multicolumn{1}{c}{ Existing } & & & & \\
LANSCE & 0.8 & $2.3 \times 10^{13}$ & 20 & 0.07 \\
ISIS & 0.8 & $2.5 \times 10^{13}$ & 50 & 0.2 \\
$\quad$ & & & \\
$\quad$ Proposed & & & & \\
JJP (Japan) & 3.0 & $8.0 \times 10^{13}$ & 25 & 1.0 \\
SNS (U.S.) & 1.0 & $2.1 \times 10^{14}$ & 60 & 2.0 \\
ESS (Europe) & 1.334 & $2.3 \times 10^{14}$ & 50 & 2.5 \\
\hline \hline
\end{tabular}


TABLE II. Major machine parameters for the original hybrid lattice Spallation Neutron Source ring.

\begin{tabular}{lcc}
\hline \multicolumn{1}{c}{ Quantity } & Value & Unit \\
\hline Circumference & 220.88 & $\mathrm{~m}$ \\
Average radius & 35.154 & $\mathrm{~m}$ \\
Injection energy & 1 & $\mathrm{GeV}$ \\
Extraction energy & 1 & $\mathrm{GeV}$ \\
Beam power & 2 & $\mathrm{MW}$ \\
Repetition rate per ring & 60 & $\mathrm{~Hz}$ \\
Number of protons & 2.08 & $10^{14}$ \\
Ring dipole field & 0.7406 & $\mathrm{~T}$ \\
rf harmonics & 1,2 & \\
Peak rf voltage, $h=1$ & 40 & $\mathrm{kV}$ \\
Peak rf voltage, $h=2$ & 20 & $\mathrm{kV}$ \\
Unnormalized emittance in $x$ or $y$ & & \\
$\quad$ cor. painting) & 120 & $\pi \mathrm{mm} \mathrm{mrad}$ \\
Unnormalized emittance $(99 \%)$ & & \\
$\quad$ (anticor. painting) & 160 & $\pi \mathrm{mm} \mathrm{mrad}$ \\
Betatron acceptance & 480 & $\pi \mathrm{mm} \mathrm{mrad}$ \\
Momentum acceptance $(160 \pi$ mm mrad) & \pm 2 & $\%$ \\
Momentum acceptance (zero amplitude) & \pm 3.8 & $\%$ \\
Magnetic rigidity, $B \rho$ & 5.6574 & $\mathrm{~T} \mathrm{~m}$ \\
Bending radius, $\rho$ & 7.6389 & $\mathrm{~m}$ \\
Horizontal tune & $5.8-6.8$ & \\
Vertical tune & $4.8-5.8$ & \\
Transition energy, $\gamma_{\mathrm{T}}$ & 4.95 & $\mathrm{GeV}$ \\
Horizontal natural chromaticity & -7.5 & \\
Vertical natural chromaticity & -6.3 & \\
Number of superperiods & 4 & \\
\hline \hline
\end{tabular}

This paper summarizes the low-loss design optimization for the SNS accumulator ring [8,9]. Many of the design concepts and conclusions can be applied to future highintensity facilities. Section II presents the FODO-doublet hybrid lattice. Considerations of physical and momentum acceptance are given in Sec. III. In Sec. IV, we compare injection painting scenarios and discuss injection halo control. Section V discusses the extraction layout. In Sec. VI, we address loss mechanisms, halo development, and space charge issues. Beam collimation, beam gap cleaning, and collimator design are discussed in Sec. VII. Radio frequency system design is presented in Sec. VIII. Magnet field error analysis and chromatic and resonance corrections are discussed in Secs. IX and X. Impedance and instability issues are discussed in Sec. XI. The diagnostics system is discussed in Sec. XII. The functions of the transport lines to the ring are presented in Sec. XIII. A summary is given in Sec. XIV.

\section{FODO-DOUBLET HYBRID LATTICE}

\section{A. Layout and functions}

Lattices used in typical high-intensity proton accelerators have either a FODO structure (AGS Booster [10], IPNS upgrade [11], Japan Joint Project (JJP) ring [12], previous SNS ring [5], etc.) or a doublet/triplet structure (ISIS

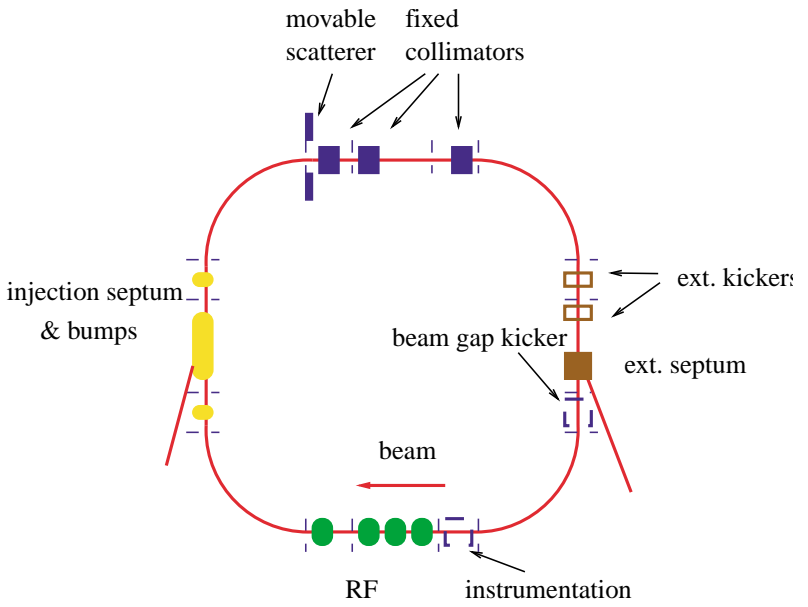

FIG. 1. (Color) Schematic layout of the SNS accumulator ring. The four straight sections are designed for beam injection, collimation, extraction, and rf systems, respectively.

[13], ESS [14], etc.). A FODO lattice structure has the advantage of relatively low quadrupole gradients, relatively smooth lattice function variations, and easily implemented chromatic and resonance corrections. However, the uninterrupted drift space is often short and not desirable for injection and collimation arrangements. Moreover, possible lattice mismatch caused by unequal FODO cell lengths can reduce machine acceptance. On the other hand, a doublet/triplet lattice structure has the advantage of long uninterrupted drift spaces for injection and collimation optimization.

The newly optimized SNS ring lattice has a hybrid structure with FODO bending arcs and doublet straight sections [6]. The lattice combines the FODO structure's simplicity and ease of correction with the doublet structure's flexibility. As shown in Fig. 1, the accumulator ring has a fourfold symmetry comprising four FODO arcs and four dispersion-free straights. The four straight sections house injection, collimation, rf, and extraction systems, respectively. Each straight section consists of one $9 \mathrm{~m}$ and two $5.5 \mathrm{~m}$ long dispersion-free drifts.

Figure 2 shows the layout and content of one of the four superperiods. Each arc consists of four $8 \mathrm{~m}$ long FODO cells. Five of the arc quadrupoles, at sites of large dispersion, are sandwiched by a chromatic sextupole and an orbit correction dipole. The other quadrupoles are sandwiched by corrector packages containing both linear elements for orbit correction and decoupling and nonlinear elements for amplitude detuning and resonance correction.

\section{B. Lattice functions and matching}

Figure 3 shows the lattice functions in one lattice superperiod, consisting of a FODO arc and a doublet straight. The arcs and straight sections are optically matched to ensure maximum betatron acceptance. A horizontal betatron phase advance of $2 \pi$ rad across each arc makes each arc 


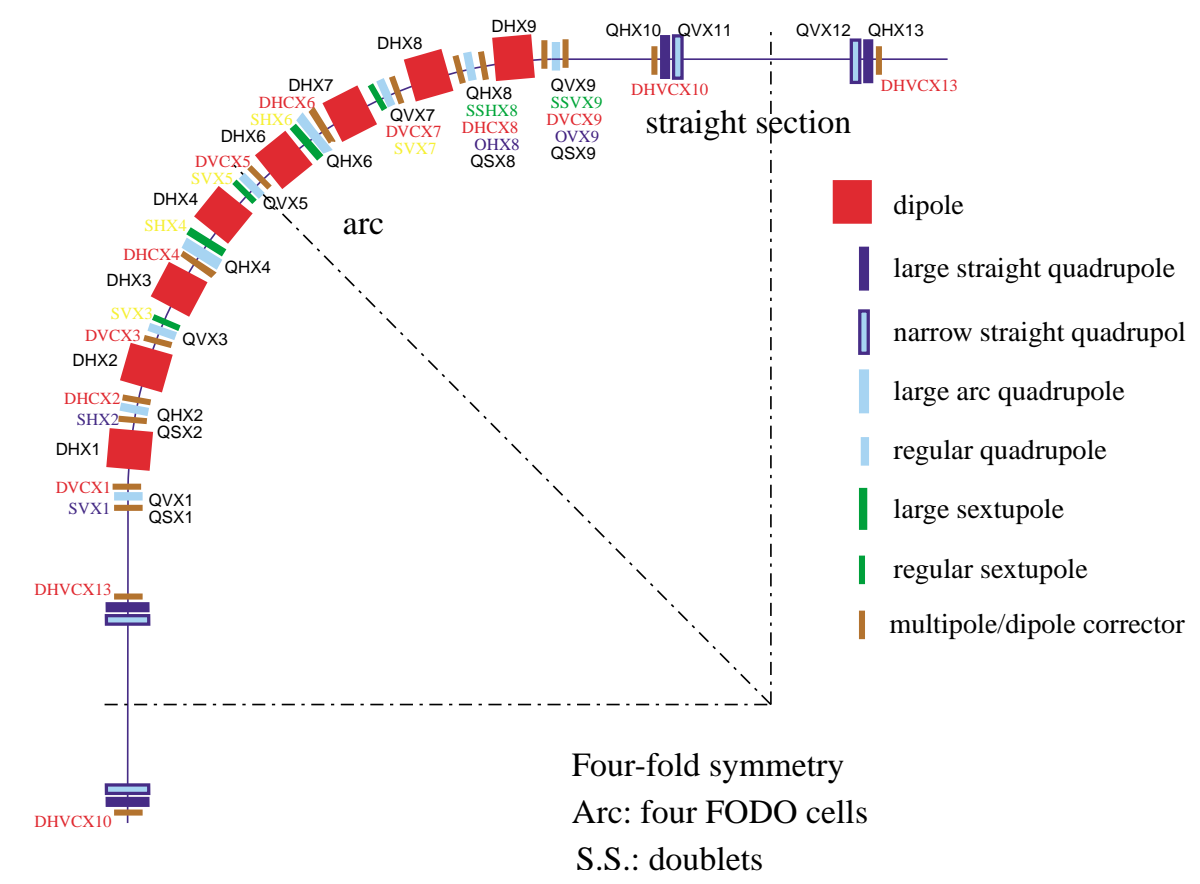

FIG. 2. (Color) Schematic layout showing dipole, quadrupole, sextupole, and corrector magnets of one lattice superperiod.

an achromat. The dispersion vanishes in the straight sections. Each dipole is centered between two quadrupoles so as to maximize the vertical acceptance of the dipoles.

\section{Working points}

The horizontal and vertical tunes can both be adjusted by more than one unit without significant optical mismatch. The vertical tune is adjusted using the two families of arc quadrupoles, and the horizontal tune is adjusted using the three families of straight-section quadrupoles.

Working points in tune space are chosen mainly to avoid the major low-order structure resonances. Working points with tunes split by more than a half-integer avoid possible strong coupling caused by space-charge forces and systematic magnet errors, thus preserving the painted beam distribution for the target. Table III compares four candidates

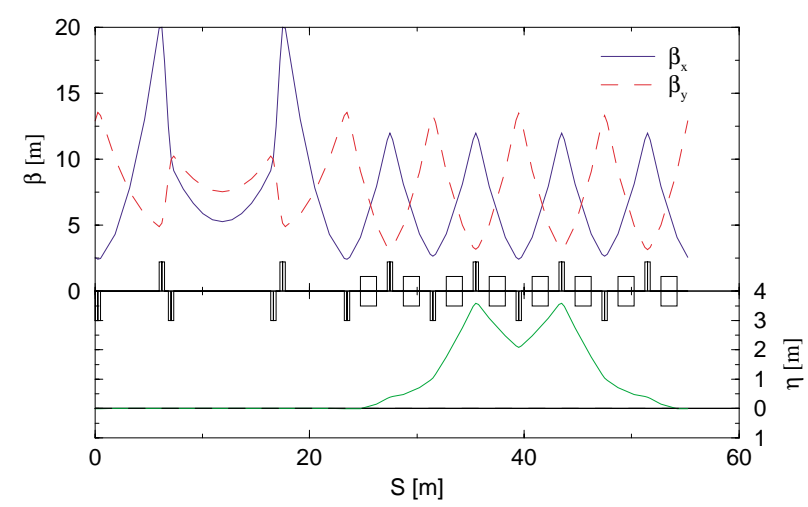

FIG. 3. (Color) Lattice functions of one lattice superperiod consisting of a FODO arc and a doublet straight. The horizontal phase advance across the arc section is $2 \pi \mathrm{rad}$. The dispersion in the straight section is zero. in $\left(Q_{x}, Q_{y}\right)$ tune space. A more detailed working-point comparison based on resonance analysis is currently under study.

\section{Alternative lattices}

Several alternative lattices have been studied and compared with the nominal lattice. One alternative reverses the polarity of the quadrupoles. In that case, the maximum dispersion is increased by about $10 \%$. Another alternative [11] uses missing-dipole dispersion suppressors, instead of the achromats, to reduce dispersion in the straights. In this case, dispersion matching can reduce the maximum dispersion of the ring by about $40 \%$, and the horizontal phase advance in the arc is more flexible. However, the addition of eight FODO half-cells with missing dipoles increases the ring circumference by about $15 \%$.

TABLE III. Comparison of SNS ring working points in the tune space.

\begin{tabular}{|c|c|c|}
\hline$\left(Q_{x}, Q_{y}\right)$ & Advantages & Disadvantages \\
\hline$(6.30,5.80)$ & $\begin{array}{l}\text { perfect matching } \\
\text { split tune } \\
\text { high tunes }\end{array}$ & $\begin{array}{l}\text { near } 2 Q_{x}+2 Q_{y}=24 \\
\text { near } 2 Q_{x}=12\end{array}$ \\
\hline$(6.30,5.27)$ & $\begin{array}{l}\text { perfect matching } \\
\text { split tune }\end{array}$ & $\begin{array}{l}\text { near } 3 Q_{y}=16 \\
\text { near } 2 Q_{y}-Q_{x}=4 \\
\text { near } 2 Q_{x}=12\end{array}$ \\
\hline$(5.82,5.80)$ & $\begin{array}{l}\text { coupled painting } \\
\text { away from integer }\end{array}$ & $\begin{array}{l}\text { large } \beta_{\max } / \beta_{\min } \\
\text { coupling \& growth } \\
\text { near } 2 Q_{x}-2 Q_{y}=0\end{array}$ \\
\hline$(5.82,4.80)$ & $\begin{array}{l}\text { split tune } \\
\text { away from integer }\end{array}$ & $\begin{array}{l}\text { large } \beta_{\max } / \beta_{\min } \\
\text { near } 2 Q_{y}-Q_{x}=4\end{array}$ \\
\hline
\end{tabular}




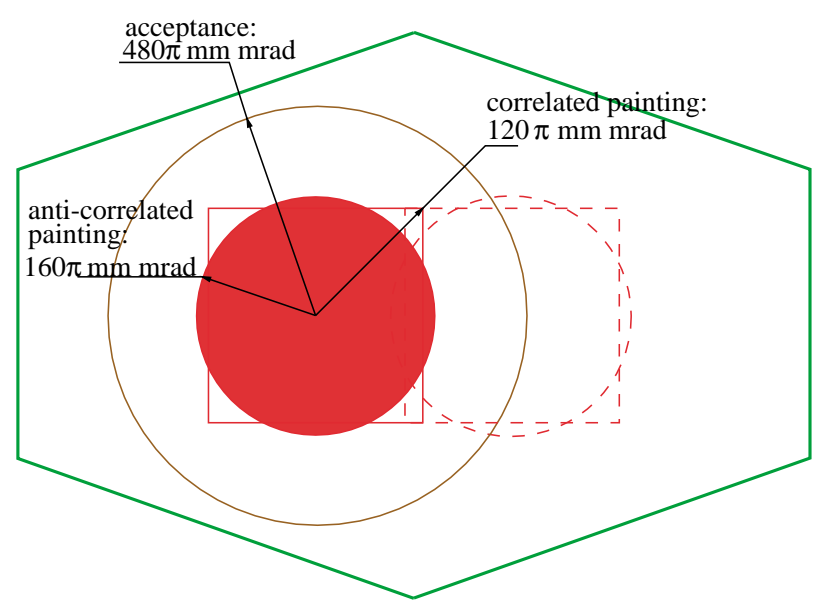

FIG. 4. (Color) Schematic illustration of the transverse dimensions in the ring. The green hexagonal boundary represents the cross section of the dipole vacuum chamber. The brown circular line corresponds to the minimum aperture in the ring at $480 \pi \mathrm{mm}$ mrad. The beam is represented by the red circle for anticorrelated painting and the red square for correlated painting. The displacement of the beam centroid in the dipole (indicated by the dashed outlines) corresponds to $1 \%$ momentum deviation.

\section{PHYSICAL AND MOMENTUM ACCEPTANCE}

An adequate acceptance-to-emittance ratio is essential to allow for collimating the beam and minimizing uncontrolled beam loss. Collimation studies indicate that efficient cleaning of a beam extending out to $240 \pi \mathrm{mm} \mathrm{mrad}$ (as in correlated painting where we paint to a square beam with emittances of $120 \pi$ in both $x$ and $y$ directions) requires a ring acceptance of $480 \pi \mathrm{mm}$ mrad. This accep- tance will allow particles scattered by the collimation system to return to the collimators without being lost elsewhere in the ring. When anticorrelated painting is used (where we paint to a circular beam), the beam extends to just $160 \pi \mathrm{mm} \mathrm{mrad}$, and the acceptance-to-emittance ratio becomes larger (now 3 instead of 2), making collimation more efficient.

We therefore require a minimum ring aperture of $480 \pi \mathrm{mmmrad}$ everywhere around the ring. Each element is designed to provide the necessary aperture, taking into account the optic functions. Along the arcs, where the dispersion is nonzero, acceptance depends on momentum deviation. And there we require the same acceptance of $480 \pi \mathrm{mm}$ mrad for particles with momentum deviation $\delta p / p_{0}= \pm 1 \%$. This corresponds to the rf bucket acceptance. Figure 4 illustrates schematically the transverse dimensions of the beam, collimators, and beam pipe around the ring.

Finally, to allow for longitudinal collimation with the beam-in-gap kicker (see Sec. VII), we require the longitudinal acceptance of the arc to be $\delta p / p_{0}= \pm 2 \%$ for a beam extending out to $160 \pi \mathrm{mm}$ mrad. Then offmomentum particles can reach the gap between bunches without hitting the vacuum chamber at high-dispersion areas. This longitudinal acceptance will allow one to make chromaticity measurements and upgrade the rf to cure instabilities.

\section{INJECTION}

The $\mathrm{H}^{-}$beam is transferred from the linac, stripped by the foil, and then injected into the ring. A maximum field

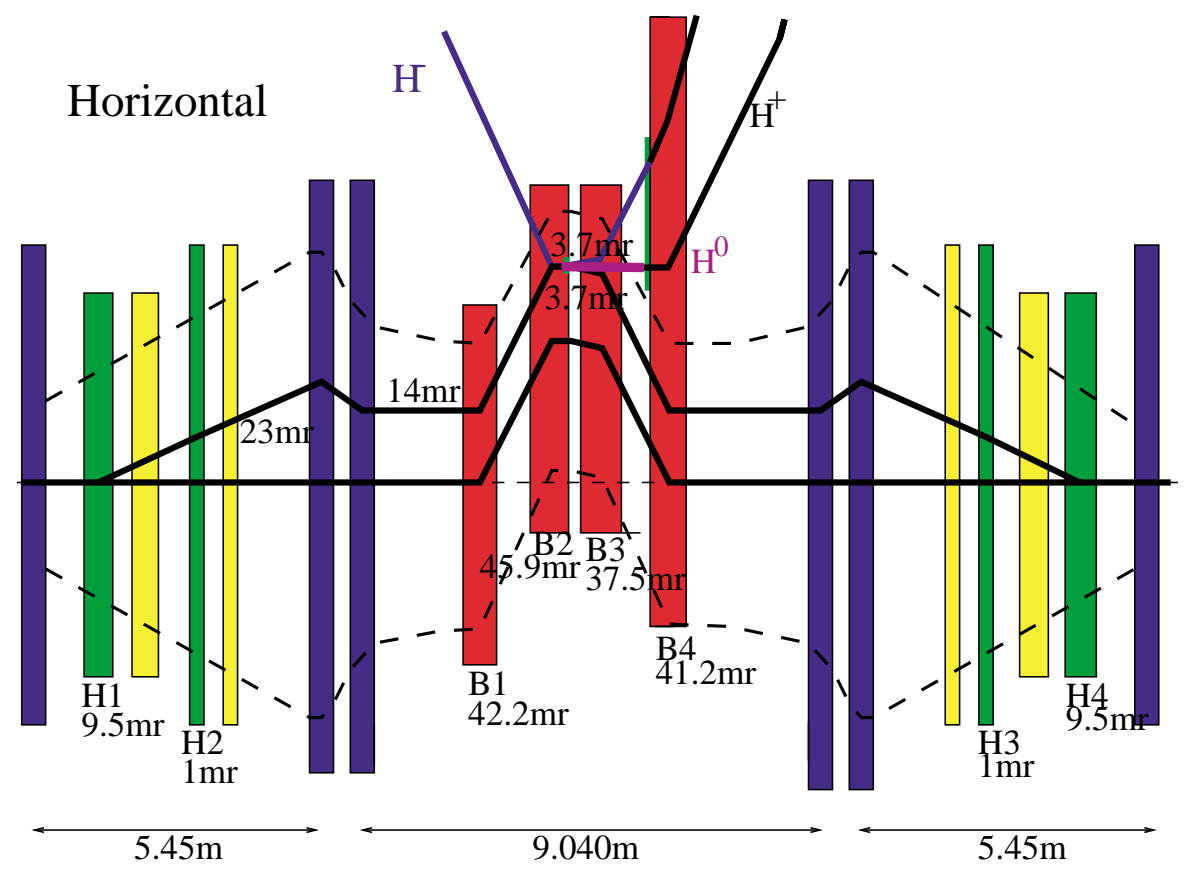

FIG. 5. (Color) Schematic layout of the injection straight section. The red elements are the fixed injection chicane, the blue elements are ring lattice quadrupoles, and the yellow and green elements are the vertical and horizontal dynamic kickers, respectively. 


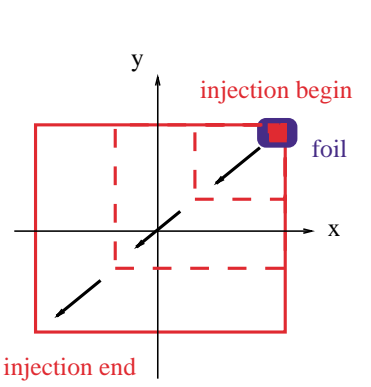

(a)

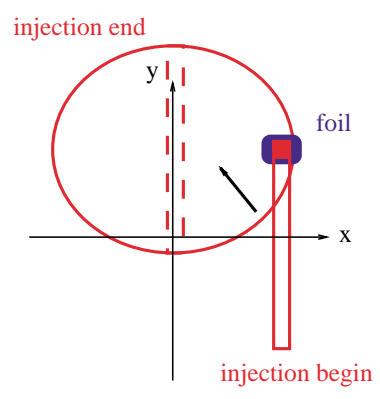

(b)
FIG. 6. (Color) Two possible painting schemes: (a) correlated bumps yield a rectangular beam profile and (b) anticorrelated bumps yield an elliptical beam. The beam is injected on a fixed location of the stripping foil.

of $0.3 \mathrm{~T}$ in the transport line and injection magnet keeps premature $\mathrm{H}^{-}$stripping below $10^{-6}$ per meter.

\section{A. Injection layout}

As shown in Fig. 5, a $9 \mathrm{~m}$ drift between quadrupole doublets houses the fixed chicane that assures adequate clearance for injection. To prevent stripping of $\mathrm{H}^{0}$ in $n=$ 4 and lower excited states, the injection stripping foil is located at the downstream end of the injection dipole, and the field of the subsequent dipole magnet is $2.4 \mathrm{kG}$. The fringe field of the injection dipole is shaped so that stripped electrons spiral down to where they can be easily collected.
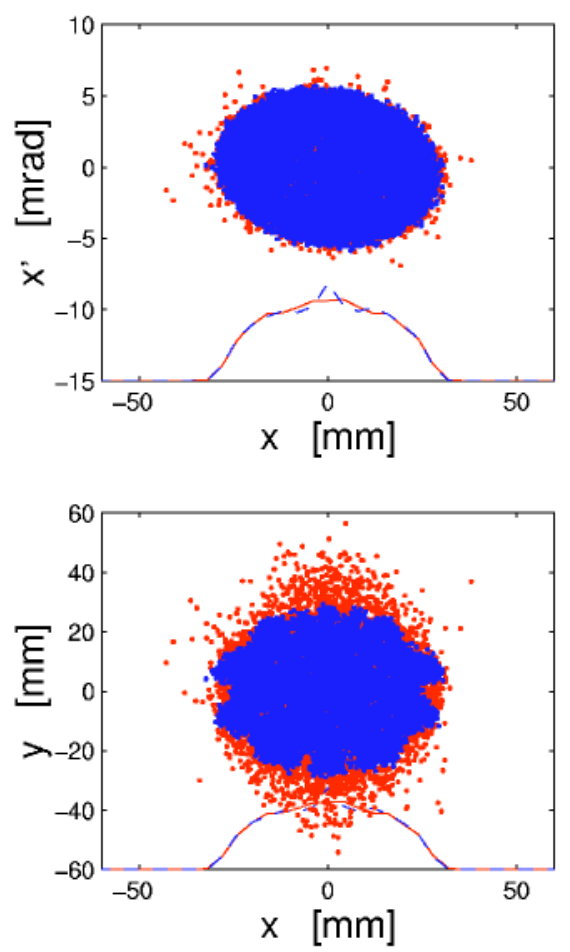

The two $5.5 \mathrm{~m}$ drifts accommodate the symmetrically placed horizontal and vertical dynamic kickers used for injection painting. The $\beta$-function perturbation caused by the injection chicane and the orbit bumps is about $2 \%$. The maximum residual dispersion is about $0.2 \mathrm{~m}$. The amplitude dependent tune shift produced by the chicane (0.004) is small compared with that produced by spacecharge forces $(0.15$; see Table VII).

The fixed chicane does not overlap ring lattice magnets. When the lattice is tuned, the strengths of the dynamic kickers will be adjusted so that the fixed chicane remains constant. The injection system is thus decoupled from the lattice tuning.

\section{B. Painting scheme comparison}

Injection painting creates the transverse density and beam profile desired at the mercury target. In addition, the painting is designed to achieve an average of less than eight foil hits per particle during the full 1225-turn accumulation. Various painting schemes have been explored: correlated, anticorrelated [15], and transverse coupled painting (see Fig. 6). Particle-in-cell simulations, using the codes SIMPSONS and ORBIT, were performed with beams having unnormalized rms emittances of about $0.25 \pi \mathrm{mm} \mathrm{mrad}$, but different distributions. However, because of the very small injected phase-space area, the difference in injected distributions has little effect on the final beam distribution.
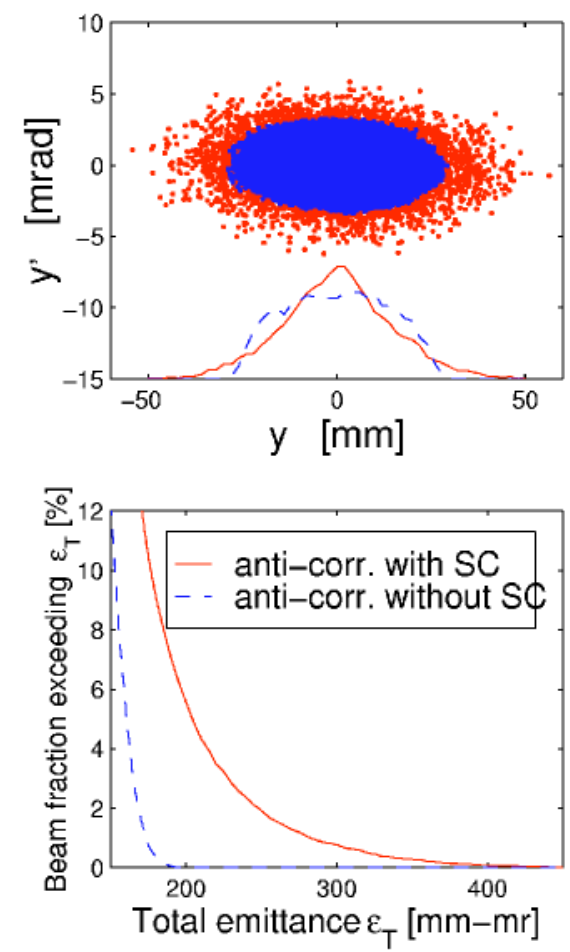

FIG. 7. (Color) Vertical emittance growth in anticorrelated painting (tunes: 6.3, 5.8). For the data shown in blue, space charge was neglected; for the data shown in red, the space charge force for a $2 \mathrm{MW}$ beam was included. Space charge produces a significant beam tail. 

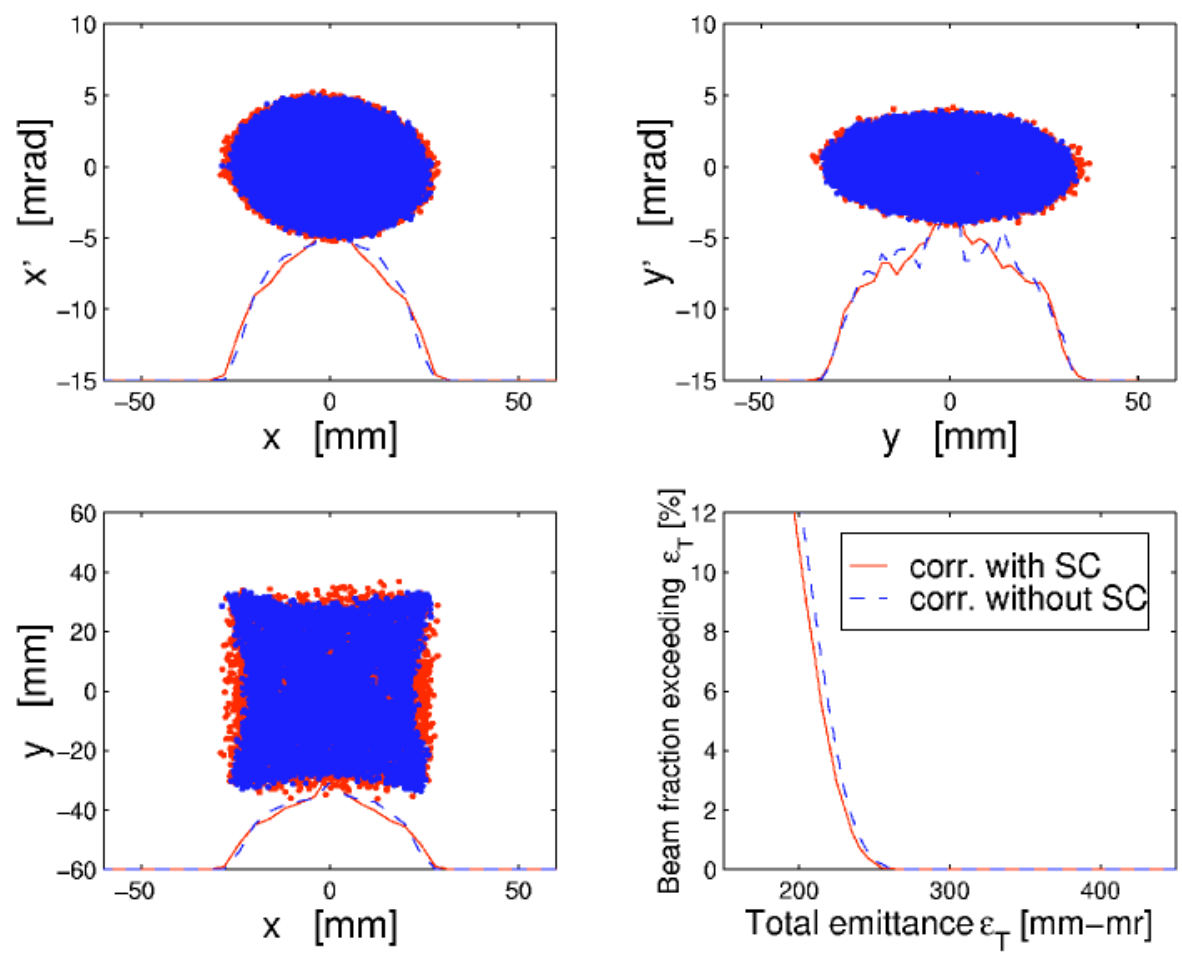

FIG. 8. (Color) Rectangular transverse beam profile resulted from correlated painting (tunes: 6.3, 5.8). The color code is the same as in Fig. 7. Here, space charge produces an insignificant beam tail.

Ideally, anticorrelated painting using opposite horizontal and vertical orbit bumps produces a distribution with an elliptical transverse profile and a uniform density distribution. Such a distribution can also be realized by painting in one direction and steering in the other. However, in the presence of space charge this scheme produces an excessive beam halo during the early stage of painting, when the beam is narrow in one direction (see Fig. 7). Further analysis indicates that this beam halo can be reduced by a new anticorrelated painting: alternating anticorrelated orbit bumps in both the horizontal and vertical directions.

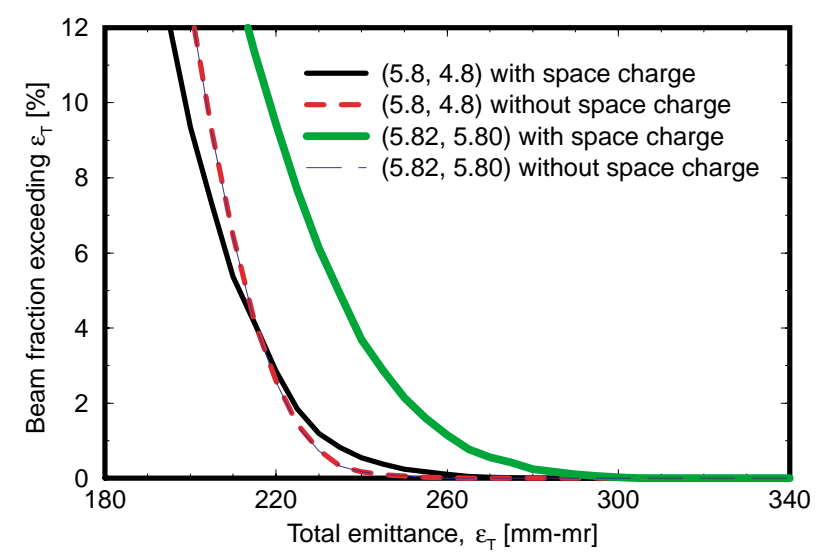

FIG. 9. (Color) Comparison of beam tail growth between same tune and split-tune working points (correlated painting). A horizontal-vertical tune split significantly suppresses beam-tail growth caused by space-charge coupling.
Correlated painting using parallel horizontal and vertical orbit bumps produces a rectangular transverse profile. Figure 8 shows the beam distribution in the case of the half-integer split tune working point $(6.30,5.80)$. This scheme has the advantage that the beam halo is constantly painted over by freshly injected beam. The main concern is whether the rectangular beam profile can be preserved in the presence of coupling produced by space charge and

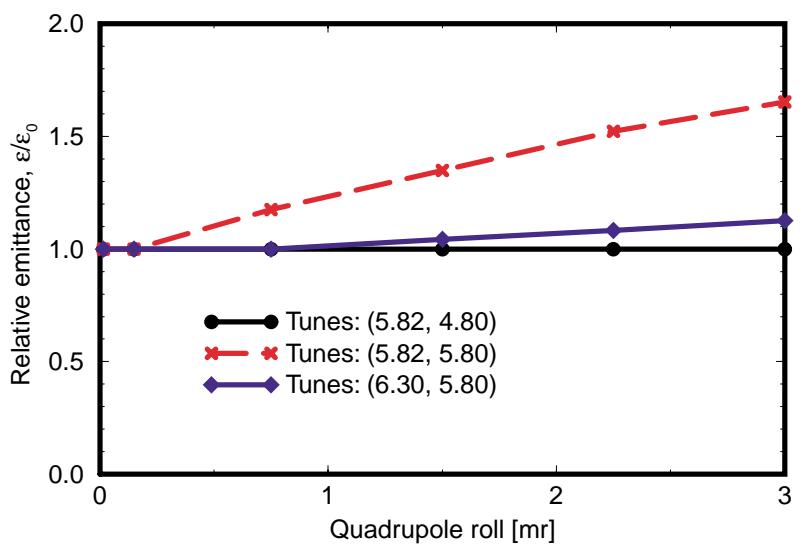

FIG. 10. (Color) Growth in $99.9 \%$ emittance (without space charge) as a function of systematic quadrupole roll for unsplit tune, half-integer split tune, and integer split-tune working point. Only one quadrupole per lattice superperiod is rolled. A horizontal-vertical tune split significantly suppresses emittance growth caused by quadrupole misalignment. The actual expected quadrupole roll in the SNS is in the range from 0.2 to $1 \mathrm{mrad}$ randomly distributed. 
magnet errors. Figure 9 compares the beam tail development between cases with and without full-integer tune split. Obviously, splitting the transverse tunes greatly reduces space-charge induced beam tail and helps preserve the rectangular beam shape. Also, as shown in Fig. 10 (without space charge), splitting of the tunes can reduce the impact of a systematic skew quadrupole component induced, for example, by quadrupole rolls.

\section{EXTRACTION}

The beam accumulated in the SNS ring forms a single $590 \mathrm{~ns}$ long bunch with a gap of $250 \mathrm{~ns}$. The extraction system consists of 14 fast kickers and a single Lambertson septum (see Fig. 11). Extraction is a two-step process: kick the beam vertically with the fast kickers into the Lambertson-type septum magnet, then use the septum magnet to deflect the beam horizontally.

In most machines, the extraction region has a high radioactivation level resulting from accidental beam loss caused by missteering and kicker malfunction. The SNS ring extraction system is designed to accept the fully painted beam without loss even when one of the 14 kickers fails. The acceptance of the extraction channel is $400 \pi \mathrm{mm}$ mrad, as compared to the ring acceptance of $480 \pi \mathrm{mm}$ mrad. To achieve a fractional beam loss below $10^{-6}$ at the extraction channel under normal operating conditions, the beam is collimated for an extra 20 turns after accumulation.

\section{HALO AND SPACE CHARGE}

\section{A. Beam tail and halo development}

Space-charge forces and magnet field errors can drive particles into resonance, resulting in emittance increase

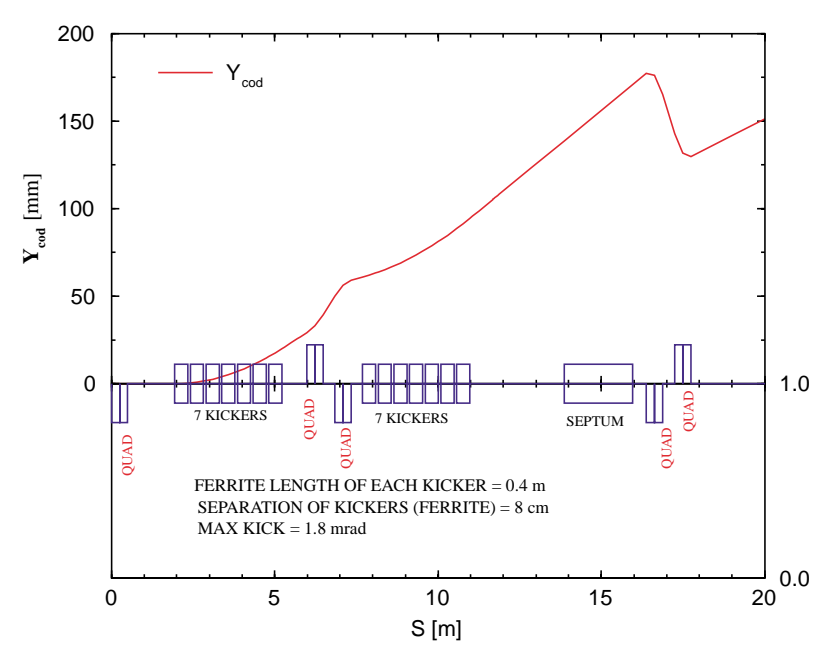

FIG. 11. (Color) Ring extraction layout and orbit. The beam is kicked vertically by 14 kicker modules and extracted horizontally by a Lambertson septum magnet.

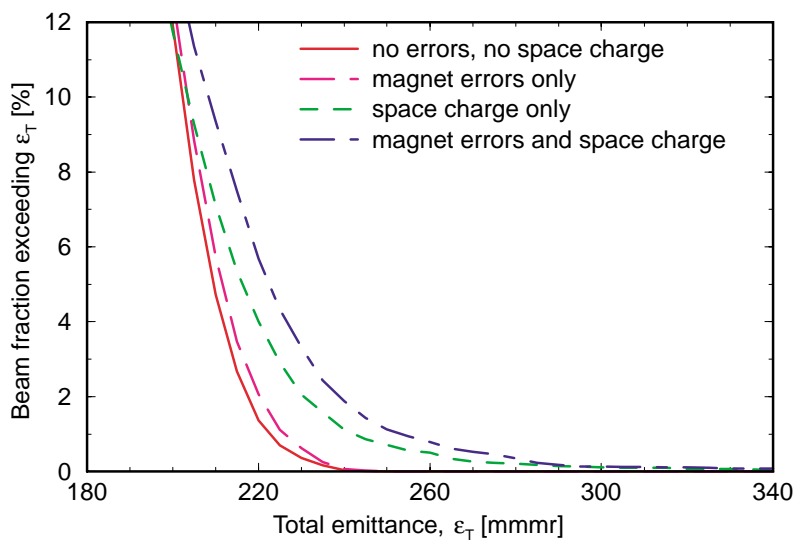

FIG. 12. (Color) Beam tail driven by space charge and magnet errors. The development of beam tail is noticeably enhanced by the combination of these two driving sources. The same-tune working point $(5.82,5.80)$ is chosen to illustrate the effect.

and particle loss. Figure 12 presents SIMPSONS [16] simulation results showing the beam tail developed in the presence of space charge and magnet errors when operating at the same-tune working point $(5.82,5.80)$. At this working point, we are very close to a space-charge induced coupling resonance $2 Q_{x}-2 Q_{y}=0$. Although difference resonances are not considered dangerous to the operation of accelerators, this observed coupling resonance leads to significant beam tail growth which is undesirable for the low-loss design. The present lattice design allows one to choose different working points in a tune range of more than one unit. Clearly, the beam tail generated by the coupling resonance can be significantly reduced when a split-tune working point is chosen (recall Fig. 9). These results have been independently confirmed using ORBIT codes [17] and UAL packages [18].

\section{B. Parametric halo in ring}

A 1:2 parametric resonance is believed to be the principal mechanism behind space-charge induced halos in linacs (see the extensive literature listed in [19]). This resonance between the motion of individual ions and collective beam oscillations is governed primarily by the rms beam mismatch. The dynamics in ring is quite different. In the low space-charge regime typical of high-intensity rings with tune depressions of a few percent, the transverse particle motion near the core of the beam is regular. The rate at which particles are trapped by the 1:2 resonance becomes very small. In addition, in proposed high-intensity rings, because of the multiturn injection painting, the final intensity is reached only at the end of the injection process, and almost immediate extraction leaves little time for a parametric halo to develop. Furthermore, because of the multiturn injection painting, the mismatched coherent modes of the beam may be damped by phase mixing. Hence, for halo development in the SNS ring, mechanisms besides parametric resonance may be more important [20]. 


\section{Effective space-charge tune shift}

Space charge is a fundamental limitation in highintensity circular accelerators. Rings are usually designed to avoid strong resonances using a formula for the incoherent space-charge tune shift which is based on the assumption of a constant beam size. Taking into account the oscillation of the beam envelope $[21,22]$, the resonance condition is actually [23]

$$
\frac{n}{m}=Q_{0}-C_{m} \Delta Q_{\mathrm{sc}},
$$

where $Q_{0}$ is the base tune, $\Delta Q_{\mathrm{sc}}$ is the incoherent spacecharge tune shift, $n$ is the excited harmonic, $m$ is the resonance order, and deviation of the coefficient $C_{m}$ from 1 represents the contribution of envelope oscillations. This contribution is most significant for low-order resonances $[24,25]$. For a round beam near a half-integer resonance $(m=2)$, when the horizontal and vertical tunes are close to each other, $C_{2}=1 / 2$ for the symmetric mode, and $C_{2}=3 / 4$ for the antisymmetric mode. In the case of split tunes, $C_{2}=5 / 8$. For the SNS ring at the split-tune working point with $\Delta Q_{\mathrm{sc}} \approx 0.15$, the effective tune shift is $\Delta Q_{\text {eff }}=5 \Delta Q_{\text {sc }} / 8=0.09$. The actual space-charge limit is therefore less restrictive.

\section{BEAM COLLIMATION}

The incoming linac halo is cleaned by the linac-toring transport line collimation system, which consists of adjustable stripping foils for $\mathrm{H}^{-}$particles followed by shielded collection elements. The linac's energy tail is cleaned by a momentum collimation system located in the maximum dispersion region of the transport line's achromat bend. In the ring, an entire straight section is dedicated to a two-stage collimation system. A beam-in-gap kicker is used to clean the residual in the gap between subsequent beam pulses.

\section{A. Transverse collimation}

The machine is designed to localize beam losses to shielded locations using beam collimators [26,27]. Ring collimation employs a two-stage system located in a shielded nondispersive straight section. The adjustable primary scraper, shielded by a collimation block, is used to intercept incoming halo and can also be used for beam diagnostics. Two secondary collimators, located at an acceptance of about $300 \pi \mathrm{mm} \mathrm{mrad}$, are designed to catch particles scattered by the scraper. Their phase advances relative to the primary collimator are determined from efficiency optimization and lattice constraints.

Figure 13 compares the collimation inefficiency of the previous all-FODO lattice with that of the hybrid lattice. With the long drift space provided by the hybrid lattice, the collimators can now be arranged at locations of optimum betatron phase to enhance the efficiency. We expect the

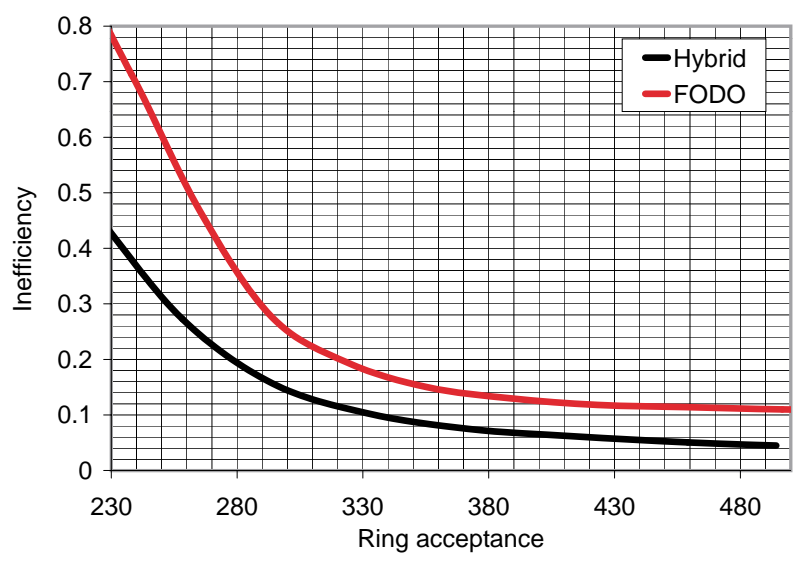

FIG. 13. (Color) Comparison of collimation inefficiency between the previous all-FODO lattice (upper curve) and the present hybrid lattice (lower curve). The inefficiency is defined as the number of halo particles escaping the collimation system after one turn above a given amplitude.

collimation efficiency to be around $90 \%$. The secondary collimation units, comprising layers of steel, borated water, and the like, are designed to contain secondary charged and uncharged particles [28], as shown in Fig. 14. Because of stringent engineering requirements for stress, heat tolerance (about $1 \mathrm{~kW}$ power), and shielding, these units are not adjustable. Based on the results of a recent experiment at the BNL Tandem [29], a serrated coated surface will be used to minimize secondary electron emission.

\section{B. Momentum and beam-in-gap cleaning}

Various mechanisms, including chopper inefficiency and foil ionization, can produce a residual beam between microbunches, resulting in uncontrolled loss at extraction. A gap-cleaning kicker is designed to resonantly excite coherent betatron oscillations, driving the gap beam into the primary collimator, where beam loss is measured with a gated fast loss monitor [30]. The hardware is similar to that of the RHIC damper/tune monitor system, which uses commercially available MOSFET banks to supply $5 \mathrm{kV}, 120 \mathrm{~A}, 10 \mathrm{~ns}$ rise and fall time pulses to a transmission line kicker. The burst mode frequency, $<1 \mathrm{MHz}$, will permit turn-by-turn kicking. With a kicker length of $5 \mathrm{~m}$, the gap can be cleaned in about 20 turns.

\section{RADIO-FREQUENCY SYSTEMS}

The main purpose of the rf system is to maintain a $250 \mathrm{~ns}$ gap for the rise of the extraction kicker. It will also (i) control the peak beam current to prevent space charge stop-band related losses and (ii) maintain a large momentum spread to prevent coherent instabilities. This momentum spread will also Landau damp coherent quadrupole oscillations which can drive halo formation. Compared with a single harmonic rf, a dual rf system has significant advantages. A barrier bucket rf system may be even better, but issues such as beam loading still need to be resolved. 


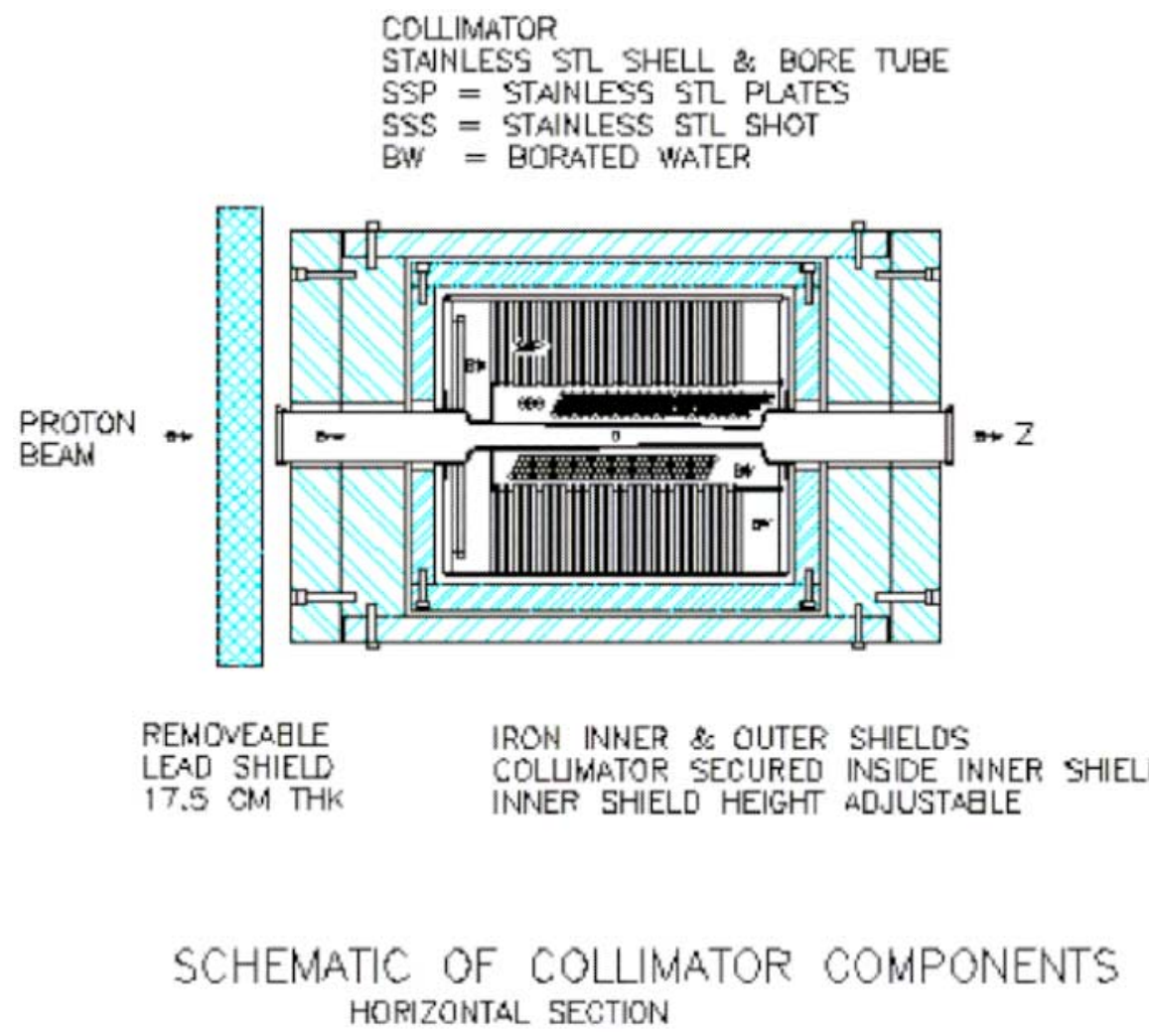

FIG. 14. (Color) Schematic of the SNS ring collimator showing layers of material for radioactivation containment.

The SNS ring will have a dual harmonic system with peak rf amplitudes of $40 \mathrm{kV}$ for harmonic $h=1$ and $20 \mathrm{kV}$ for $h=2$. Canonically, the voltages are phased so that the small amplitude synchrotron frequency vanishes. The design of the rf system and power amplifier are driven by beam loading requirements. The power amplifier is designed to fully compensate the beam current while providing the quadrature component to drive the gap voltage. As the beam is accumulated in the ring, a feed-forward system will adjust the input into the low-level rf drive.

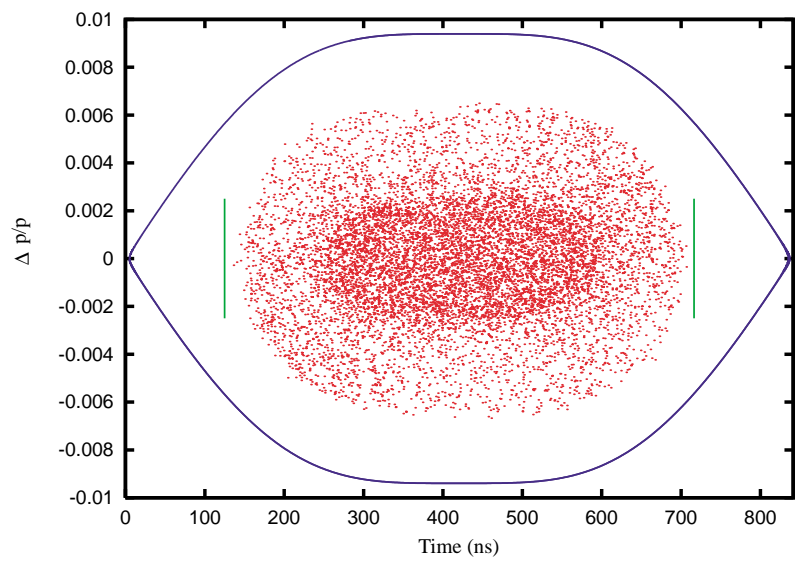

FIG. 15. (Color) Longitudinal phase space at the end of 2 MW beam accumulation. The blue curve outlines the rf bucket. The vertical lines delineate the edges of a $250 \mathrm{~ns}$ gap. The effects of space charge and cavity beam loading are included.
Shown in Fig. 15 are the results of a simulation using a linac bunch length of $546 \mathrm{~ns}$ with the first harmonic voltage ramped from 30 to $40 \mathrm{kV}$ over the first 500 turns. The amplitude of the second harmonic was half the amplitude of the first for the entire simulation, which included beam loading and longitudinal space charge. The rf was corrected using both feed forward and low level loops. We note that the full momentum spread $(\Delta p / p)$ of the incoming linac beam, after the energy-spreading cavity, is $\pm 0.26 \%$. Also, at the end of injection the beam bunching factor is approximately 0.46 .

\section{MAGNET FIELD ERROR ANALYSIS}

\section{A. Expected fringe field errors and compensation}

The bore of the SNS ring magnets is necessarily large to provide the required acceptance. The quadrupole aspect ratio, inner diameter over magnetic length, is about 0.5 . With such a high aspect ratio, contributions from the magnet ends are significant. Table IV indicates that, in the absence of pole-tip shimming, the error of the first allowed multipole (dodecapole, $b_{5}$ ) in the quadrupole magnet is exceedingly large. Multipole contributions from the ends of the unshimmed dipole include a similarly large sextupole component.

With detailed pole-tip compensation, the integral field error can be greatly reduced. Table V shows the expected magnetic error of the ring quadrupoles based on measure- 
TABLE IV. Integrated quadrupole end field from one magnet end before pole tip end shimming, extracted from a 3D TOSCA calculation (normalized to $10^{-4}$ of the main field at the reference radius $R_{\text {ref }}$ ). For regular ring quadrupoles, $R_{\text {ref }}=10 \mathrm{~cm}$; for large ring quadrupoles, $R_{\text {ref }}=12 \mathrm{~cm}$ (approximately $92 \%$ of the quadrupole iron pole tip radius).

\begin{tabular}{ccccc}
\hline \hline & \multicolumn{3}{c}{ Normal } & \multicolumn{2}{c}{ Skew } \\
$n$ & $\left\langle b_{n}\right\rangle$ & $\sigma\left(b_{n}\right)$ & $\left\langle a_{n}\right\rangle$ & $\sigma\left(a_{n}\right)$ \\
\hline 2 & 0.4 & $\ldots$ & 0.0 & $\ldots$ \\
3 & 0.1 & $\ldots$ & 0.0 & $\ldots$ \\
4 & 0.7 & $\ldots$ & 0.0 & $\cdots$ \\
5 & 121 & $\ldots$ & 0.0 & $\cdots$ \\
\hline \hline
\end{tabular}

TABLE V. Expected magnetic errors of ring quadrupoles. The multipole strengths are normalized to $10^{-4}$ of the main field at the reference radius $R_{\text {ref }}$.

\begin{tabular}{ccccc}
\hline \hline & \multicolumn{2}{c}{ Normal } & \multicolumn{2}{c}{ Skew } \\
$n$ & $\left\langle b_{n}\right\rangle$ & $\sigma\left(b_{n}\right)$ & $\left\langle a_{n}\right\rangle$ & $\sigma\left(a_{n}\right)$ \\
\hline Body & [unit] & & & \\
2 & 0.0 & -2.46 & 0.0 & -2.5 \\
3 & 0.0 & -0.76 & 0.0 & -2.0 \\
4 & 0.0 & -0.63 & 0.0 & 1.29 \\
5 & 0.20 & 0.0 & 0.0 & 1.45 \\
6 & 0.0 & 0.02 & 0.0 & 0.25 \\
7 & 0.0 & -0.63 & 0.0 & 0.31 \\
8 & 0.0 & 0.17 & 0.0 & -0.11 \\
9 & 0.70 & 0.0 & 0.0 & 1.04 \\
\hline \hline
\end{tabular}

TABLE VI. Expected alignment errors of ring magnets based on the survey measurement of the AGS Booster magnets and the AGS-to-RHIC transfer line magnets.

\begin{tabular}{lc}
\hline \hline \multicolumn{1}{c}{ Item } & Value \\
\hline Integral field variation (rms) & $10^{-4}$ \\
Integral field, transverse variation (rms) & $10^{-4}$ \\
Ring dipole sagitta deviation & $3 \mathrm{~cm}$ \\
Magnetic center position (rms) & $0.1-0.5 \mathrm{~mm}$ \\
Magnet longitudinal position (rms) & $0.5 \mathrm{~mm}$ \\
Mean field roll angle (rms) & $0.2-1 \mathrm{mrad}$ \\
\hline \hline
\end{tabular}

ment data from the AGS Booster magnets. Table VI shows the expected magnet misalignment errors.

The effect of the magnet fringe fields, which is often negligible in high-energy low-aspect-ratio machines (e.g. RHIC, LHC), is important for a large-acceptance high aspect ratio ring such as the SNS. Indeed, the relative impact of the longitudinal fringe field on a particle's transverse momentum is proportional to the ratio of the transverse emittance to the effective magnetic length [31]. For the lattice quadrupoles, the octupolelike transverse kick induced by this edge effect [32] has been evaluated, and its dynamic effect is quite large (see the next subsection). A flexible correction scheme with three families of octupole correctors [33] has been proposed in order to compensate the fringe field and other residual octupolelike effects.
TABLE VII. Tune spread produced by various mechanisms on a 2 MW beam with transverse emittance of $480 \pi \mathrm{mm}$ mrad and momentum spread of $\pm 1 \%$.

\begin{tabular}{lc}
\hline \hline \multicolumn{1}{c}{ Mechanism } & Full tune spread \\
\hline Space charge & $0.15-0.2(2 \mathrm{MW}$ beam $)$ \\
Chromaticity & $\pm 0.08(1 \% \Delta p / p)$ \\
Kinematic nonlinearity $(480 \pi)$ & 0.001 \\
Fringe field $(480 \pi)$ & 0.025 \\
Uncompensated ring magnet & \\
$\quad$ error $(480 \pi)$ & \pm 0.02 \\
Compensated ring magnet & \\
$\quad$ error $(480 \pi)$ & \pm 0.002 \\
Fixed injection chicane & 0.004 \\
Injection painting bump & 0.001 \\
\hline \hline
\end{tabular}

\section{B. Expected tune spreads}

Table VII lists the tune spread produced by various effects - space charge, natural chromaticity, magnet imperfection, and the like - as indicative of their relative impact on the beam.

Because of broadening of the beam momentum before injection, tune spread produced by the natural chromaticity is significant. Section $\mathrm{X}$ discusses chromatic compensation using multifamily sextupoles.

The tune spread produced by the kinematic nonlinearity - obtained by using either UAL/TEAPOT [34] or MARYLIE [35] and by analytical evaluation [36] based on perturbation theory-originates from the large $\gamma$ functions in the straight sections of the SNS ring. It is proportional to the 4th power of the particle transverse momenta (i.e., an octupolelike effect) and becomes noticeable in the SNS, which has a large-emittance beam.

Tune spreads produced by magnet fringe fields, as mentioned in the previous subsection, have been obtained with mapping, tracking, and analytical evaluation. For the mapping approach we used MARYLIE to construct a symplectic Lie map up to third order using either a hard-edge fringe field model (taking the limit of the fringe length to 0 ) or a 3D magnetic field calculation using OPERA 3D. Comparison of the two shows that the hard-edge model provides an excellent approximation of the effect. For the tracking approach we first extracted a $2 \mathrm{D}$ multipole expansion from the 3D field calculation. Then UAL/TEAPOT was used to perform turn-by-turn tracking to evaluate detuning. Comparison of the tracking approach with either mapping or analytical evaluation shows that using a 2D multipole expansion, extracted from the 3D field calculation, grossly underestimates the importance of quadrupole fringe fields. On the other hand, the tune spread produced by the dipole fringe field is negligible.

\section{Dynamic aperture}

As an example of a dynamic aperture study, Fig. 16 shows the impact of magnet field errors and the improve- 


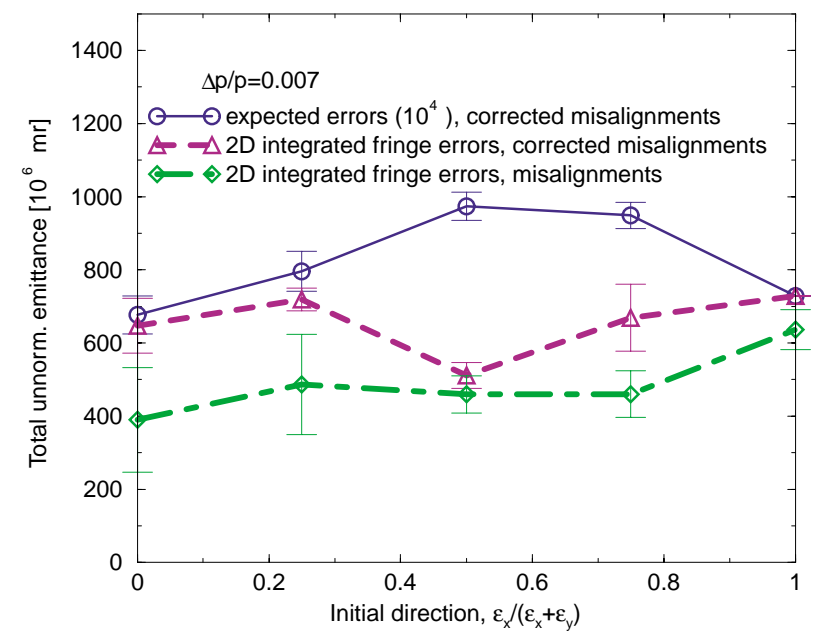

FIG. 16. (Color) Dynamic aperture obtained from 6D UAL computer tracking. Each data point gives the mean and standard deviation obtained using ten random seeds.

ment from field compensation and orbit correction. The six-dimensional element-by-element computer tracking is performed with the UAL [18] over the entire accumulation period. Initially, particles are launched at three momenta $(\Delta p / p=0, \pm 0.7 \%)$, in five transverse directions, with increasing betatron amplitude. The average dynamic aperture and the statistical errors are obtained from the results of ten random seeds. The green curve shows the combined effect resulting from the integrated 2D fringe field component of the uncompensated magnet pole tips (given in Table IV) and misalignments $(0.5 \mathrm{~mm}, 1 \mathrm{mrad})$. The violet curve indicates the sole effect of $2 \mathrm{D}$ integrated fringe field for uncompensated magnet pole tips. Finally, the blue curve represents the case of expected errors of $10^{-4}$ level (see Table V) with corrected misalignments $(0.1 \mathrm{~mm}$, $0.2 \mathrm{mrad})$.

\section{CHROMATIC AND RESONANCE CORRECTION}

Four families of chromatic sextupoles are needed to adjust the chromaticity to desired values across the beam momentum of $\pm 0.7 \%$. Figure 17 shows that, with a two-family scheme, the optical distortion in $\beta$ function is as large as $30 \%$ for off-momentum orbits. With a four-family scheme, the off-momentum optics is greatly improved (less than $2 \%$ distortion).

To compensate for field imperfections and magnet alignment errors, the ring is designed with a set of correction elements consisting of horizontal and vertical dipoles, skew quadrupoles, normal and skew sextupoles, and octupoles. Trim windings on the lattice quadrupoles allow for any necessary quadrupole corrections. The correction dipoles are mounted close to each quadrupole. Additional windings on the correction dipole cores (similar to those employed in the AGS Booster) provide four skew quadrupole and two skew sextupole correctors per lattice superperiod. The addition of two families of

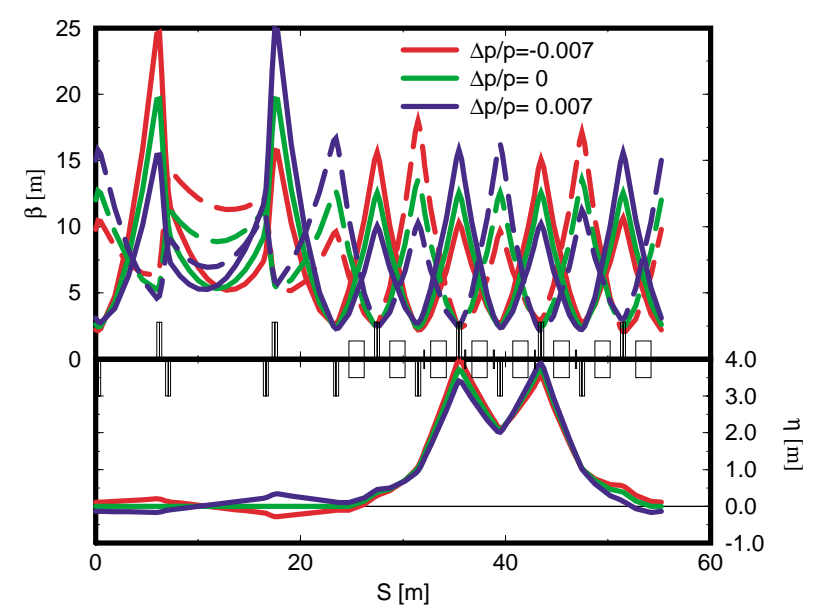

FIG. 17. (Color) Off-momentum lattice function perturbation when using a two-family chromaticity sextupole correction scheme. This perturbation reduces the available aperture for the off-momentum beam.

individual short fast trim quadrupoles is currently under consideration to allow for a small tune variation (of the order of \pm 0.1 ) from injection to extraction. Two sextupole and octupole correctors are mounted near horizontal and vertical beta maximums in each superperiod. This placement of correctors ensures that appropriate harmonics can be produced for the correction of resonances and closed-orbit distortion. An additional family of octupole correctors can be placed between the last arc quadrupole and the doublet in order to cancel the octupolelike tune shift, caused mainly by the quadrupole fringe fields [33].

\section{IMPEDANCE AND INSTABILITIES}

Because the SNS has an accumulation period of just $1 \mathrm{~ms}$, the instability of greatest concern is the so-called PSR instability [37], suspected to be caused by the electron cloud. Based on experience at the PSR, we have adopted several measures for the SNS ring to avoid the PSR instability: a larger beam momentum spread to damp instabilities, a cleaner beam gap, achieved using both a higher rf voltage and a gap-cleaning kicker, reduction of beam scraping and secondary emission using a multistage collimation, collection of electrons produced at the injection foil, and, finally, coating of the vacuum chamber's inner surface with TiN to reduce the secondary electron emission yield.

Operational experience at the AGS and its Booster has shown that the conventional formulation used for the resistive wall instability overestimates the growth rate, presumably because various Landau damping mechanisms are neglected. That formulation predicts a modest $1 \mathrm{~ms}$ growth rate for the SNS, at the end of stacking. Hence, the choice of stainless steel chamber is adequate. Experiences at the AGS, Booster, and ISIS have also shown that, for the longitudinal microwave instability, the conventional 
Keil-Schnell criterion is largely overestimated below transition. With total $40 \mathrm{kV}$ rf voltage, the beam momentum spread in the ring is about $\pm 0.7 \%$, which is more than adequate to avoid this instability. The transverse mode coupling is not likely to occur for the SNS ring, since the very long bunch cannot couple with the broadband impedance resonated at 1 to $2 \mathrm{GHz}$. Also, since the entire accumulation cycle is only one synchrotron period, a conventional head-tail-type instability is not a problem. Finally, transverse microwave instabilities are not predicted for the SNS ring. These types of instabilities have not been seen in existing low-energy proton synchrotrons, possibly because of damping effects caused by space charge.

To minimize resonance effects and impedance complications, vacuum chamber steps are tapered and bellows and ports are shielded. Low-frequency impedance is found to be dominated by the 14 window-frame extraction kicker magnets. Efforts are made to optimize the terminations to reduce their impedance. Similar kickers used in the AGS injection and the Booster extraction and dump lines have not been associated with any beam instabilities.

\section{DIAGNOSTICS AND INSTRUMENTATION}

The ring accumulation is a dynamic process during which the beam intensity increases by more than 3 orders of magnitude and the transverse beam radius increases by more than a factor of 12 . The ring diagnostics instrumentation is designed with a wide range of sensitivity and turn-by-turn capability to monitor beam intensity (beam current monitors), position (beam-position monitors), transverse and longitudinal profiles (ionization profile monitor, wall current monitor), and beam loss (loss monitor). Dual-plane beam-position monitors are installed in the critical areas, near the ring straight-section doublets and the middle of the arcs for orbit monitoring and local decoupling. Any beam residual between subsequent microbunches can be detected by a beam-in-gap monitor and removed by a beam-in-gap kicker. Electron detectors are planned to monitor the electron cloud in the vacuum chamber. The controls system is designed to immediately shut off subsequent beam pulses when a critical device failure is detected.

\section{TRANSPORT LINES}

\section{A. High energy transport line}

A $140 \mathrm{~m}$ long high energy beam transport (HEBT) line connects the linac to the accumulator ring and keeps the entire SNS facility within the desired footprint. Far more than a simple transfer line, the HEBT line plays an essential role in reducing beam loss at ring injection. It optically matches the linac to the ring. Its diagnostic systems characterize the beam and detect accidental ion source and linac

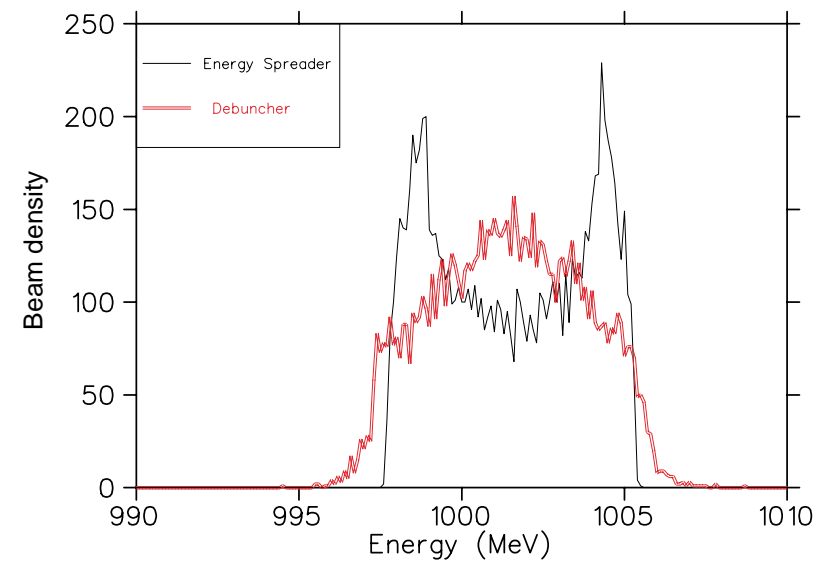

FIG. 18. (Color) Comparison of energy distributions obtained by the energy spreader and by a conventional debuncher at the injection foil. An energy spreader significantly suppresses the beam tail. Note that the distribution for the debuncher corresponds to each linac bunch, while the distribution for the energy spreader is an average over all linac bunches within the pulse.

malfunction to protect the ring. The collimation system cleans both transverse and momentum halos. The energy corrector, operating at the linac frequency, partially compensates energy jitter in the incoming linac beam. The energy spreader, operating at a phase-modulated mode of the linac frequency, provides the energy spread required for beam stability. Adjustments can be made for ground settlement and magnet misalignments. Beam-position monitors and steering magnets are designed to detect and correct closed-orbit deviations.

The HEBT line has a transverse acceptance of $30 \pi \mathrm{mm}$ mrad to accommodate an incoming beam with $0.25 \pi \mathrm{mm} \mathrm{mrad} \mathrm{rms}$ unnormalized emittance, and it has a momentum acceptance of $\pm 1 \%$ to accommodate an incoming rms momentum spread of $0.033 \%$. After the energy-spreading cavity, a total momentum spread of about $\pm 0.26 \%$ is achieved with negligible beam tail, as clearly demonstrated in Fig. 18.

\section{B. Ring to target transport line}

The $150 \mathrm{~m}$ long ring to target beam transport (RTBT) line delivers the beam to the mercury target with the required specifications. Its acceptance (transverse $480 \pi \mathrm{mm}$ mrad and momentum $\pm 1 \%$ ) accommodates one extraction kicker failure with no beam loss. The diagnostic system detects fault conditions and, together with the collimation system, protects components. Adjustments can be made for ground settlement and component misalignments, and steering magnets and beam-position monitors are designed to correct closed-orbit deviation. The betatron phase advance between the kickers and the target will be adjusted to an integer multiple of $2 \pi$ so that a kicker error does not lead to a displacement of the beam at the target. 


\section{SUMMARY}

Reliability and maintainability are of primary importance to the SNS facility. Hands-on maintenance for the accumulator ring demands an average radioactivation at or below 1-2 mSv/h, $30 \mathrm{~cm}$ from the machine device [4]. The corresponding uncontrolled fractional beam loss is about $10^{-4}$ for a $1 \mathrm{GeV}$ beam in the SNS accumulator ring.

To achieve this goal, the SNS ring design addresses the six issues that lead to heavy beam loss: (1) The beam is painted to a quasiuniform distribution to keep the space-charge tune shift below 0.15. (2) A significant transverse acceptance/emittance ratio allows the beam tail and beam halo to be cleaned by the collimation system before hitting the rest of the ring, and a stationary rf bucket confines the beam to within $70 \%$ of its momentum acceptance $(\Delta p / p= \pm 1 \%)$, while the machine's vacuum chamber provides a full momentum aperture of $\Delta p / p= \pm 2 \%$. (3) The layout and magnetic field at injection are designed to prevent premature $\mathrm{H}^{-}$and $\mathrm{H}^{0}$ stripping and excessive foil hitting. (4) A moderate main magnet field avoids saturation effects, and shimmed pole tip ends in both dipole and quadrupole magnets help compensate fringe field effects. (5) To avoid all instabilities, the vacuum chamber is coated, chamber steps are tapered, bellows and ports are shielded, and the beam momentum is broadened [38-40]. (6) The HEBT is designed against accidental ion source and linac malfunction, and the ring and RTBT are designed against accidental extraction malfunction. Significant beam loss and radio activation is expected only at the shielded collimation and injection regions.

\section{ACKNOWLEDGMENTS}

The authors would like to thank G. Rees for much valuable advice on the design, Y. Cho for discussions on the choice of doublet versus triplet straight sections, S. Machida for his assistance using the SIMPSONS codes, and many others, including J. Alessi, J. Brodowsky, J. D. Galambos, R. L. Gluckstern, H. Hseuh, D. Kaltchev, R. Kustom, J. B. Jeanneret, D. Lowenstein, A. U. Luccio, R. Macek, C. Prior, K. Reece, T. Roser, J. Sandberg, H. Schonauer, A. Soukas, R. Talman, S. Tepikian, D. Trbojevic, J. Tuozzolo, M. J. Venturini, J. G. Wang, A. Zaltsman, and the rest of the Spallation Neutron Source team.

[1] For a brief summary, see W. T. Weng, in Proceedings of the Workshop on Space Charge Physics in High Intensity Hadron Rings, edited by A. U. Luccio and W. T. Weng, AIP Conf. Proc. No. 448 (AIP, New York, 1998), p. 152.

[2] T. Wangler, RF Linear Accelerators (Wiley, New York, 1998), p. 285.

[3] T. Roser (private communication).
[4] The so-called hands-on maintenance condition corresponds to an average radiation activation level of 1 to $2 \mathrm{mSv} / \mathrm{h}$ measured at a distance of $30 \mathrm{~cm}$ from the machine device, $4 \mathrm{~h}$ after machine shutdown of an extended run of 30 days or longer. See a summary in Proceedings of the Workshop on Beam Halo and Scraping, Wisconsin, 1999 (to be published). The protection quantity Sievert (Sv) is defined as $1 \mathrm{~Sv}=100 \mathrm{rem}$ (röntgen for man). See Handbook of Accelerator Physics and Engineering, edited by A. Chao and M. Tigner (World Scientific, Singapore, 1999), p. 598.

[5] "Spallation Neutron Source Parameter List," SNS100000000-PL0001-R01, SNS Project Office, Oak Ridge, TN, 1999.

[6] Brookhaven National Laboratory, BNL/SNS Technical Note No. 66, 1999.

[7] J. Wei, J. Beebe-Wang, M. Blaskiewicz, M. Brennan, P. Cameron, N. Catalan-Lasheras, G. Danby, C. Gardner, H. C. Hseuh, J. Jackson, Y. Y. Lee, A. U. Luccio, H. Ludewig, N. Malitsky, W. Meng, D. Raparia, A. Soukas, N. Tsoupas, J. Tuozzolo, R. Witkover, A. Zaltsman, S. Y. Zhang, S. Danilov, J. D. Galambos, J. G. Wang, and G. H. Rees, in Proceedings of the 2000 European Particle Accelerator Conference, Vienna, Austria (to be published).

[8] J. Wei, J. Beebe-Wang, M. Blaskiewicz, P. Cameron, G. Danby, C. J. Gardner, J. Jackson, Y. Y. Lee, H. Ludewig, N. Malitsky, D. Raparia, N. Tsoupas, W. T. Weng, and S. Y. Zhang, in Proceedings of the 1999 Particle Accelerator Conference, New York, edited by A. Luccio and W. MacKay (IEEE, Piscataway, NJ, 1999), p. 3185.

[9] C. Gardner, Y. Y. Lee, N. Tsoupas, and J. Wei, in Proceedings of the 1999 Particle Accelerator Conference, New York (Ref. [8]), p. 3182.

[10] Booster Design Manual (Brookhaven National Laboratory, Upton, NY, 1986).

[11] Y. Cho, Y.-C. Chae, H. Friedsam, D. Horan, R. Kustom, E. Lessner, W. McDowell, D. McGhee, H. Moe, R. Nielsen, G. Norek, K. Peterson, K. Thomson, and M. White, in Proceedings of the 1996 European Particle Accelerator Conference, Sitges, Spain, 1996, edited by R. Pascual (Institute of Physics, Bristol, UK, 1996), p. 521.

[12] Y. Mori and S. Machida (private communication).

[13] Rutherford Appleton Laboratory Report No. RL 82-006, 1982, edited by B. Boardman.

[14] European Spallation Source Report No. ESS-96-53-M, 1996.

[15] J. Beebe-Wang, Y. Y. Lee, D. Raparia, and J. Wei, in Proceedings of the 1999 Particle Accelerator Conference, New York (Ref. [8]), p. 1743.

[16] S. Machida, Nucl. Instrum. Methods Phys. Res. Sect. A 309, 43 (1991).

[17] J. Galambos, J. A. Holmes, D. K. Olsen, A. Luccio, and J. Beebe-Wang, "ORBIT User's Manual” (unpublished).

[18] N. Malitsky, J. Smith, R. Talman, and J. Wei, in Proceedings of the 1999 Particle Accelerator Conference, New York (Ref. [8]), p. 2713.

[19] R. L. Gluckstern and A. V. Fedotov, in Proceedings of the Workshop on Beam Halo and Scraping, Wisconsin, 1999 (Ref. [4]).

[20] A. V. Fedotov, R. L. Gluckstern, and M. Venturini, in Proceedings of the Workshop on Beam Halo and Scraping, Wisconsin, 1999 (Ref. [4]). 
[21] L. Smith, in Proceedings of the International Conference on High Energy Accelerators, Dubna, Russia, 1963 (unpublished), p. 1232.

[22] F. Sacherer, Ph.D. thesis, University of California at Berkeley (Lawrence Radiation Laboratory Report No. UCRL18454), 1968.

[23] R. Baartman, in Proceedings of the Workshop on Space Charge Physics in High Intensity Hadron Rings (Ref. [1]), p. 56.

[24] I. Hofmann, Phys. Rev. E 57, 4713 (1998).

[25] R.L. Gluckstern, in Proceedings of the 1970 Linear Accelerator Conference, edited by M. R. Tracy (Fermilab, Batavia, IL, 1970), p. 811.

[26] N. Catalan-Lasheras, H. Ludewig, D. Raparia, and J. Wei, in Proceedings of the Workshop on Beam Halo and Scraping, Wisconsin, 1999 (Ref. [4]).

[27] N. Catalan-Lasheras, Ph.D thesis, Universidad de Zaragoza, 1999.

[28] H. Ludewig, N. Simos, J. Walker, P. Thieberger, A. Aronson, and J. Wei, in Proceedings of the 1999 Particle Accelerator Conference, New York (Ref. [8]), p. 548.

[29] P. Thieberger, A. L. Hanson, D. B. Steski, V. Zajic, S. Y. Zhang, and H. Ludewig, BNL/SNS Technical Note No. 64, 1999.
[30] R. L. Witkover, P. R. Cameron, T. J. Shea, R. C. Connolly, and M. Kesselman, in Proceedings of the 1999 Particle Accelerator Conference, New York (Ref. [8]), p. 2250.

[31] J. Wei and R. Talman, Part. Accel. 55, 339 (1996).

[32] E. Forest and J. Milutinovic, Nucl. Instrum. Methods 269, 474 (1988).

[33] Y. Papaphilippou, D. T. Abell, and C. Gardner, BNL/SNS Technical Note No. 72, 2000.

[34] L. Schachinger and R. Talman, Part. Accel. 22, 35 (1987).

[35] A. J. Dragt, D. R. Douglas, F. Neri, E. Forest, L. M. Healy, P. Schutt, and J. van Zeijts, MARYLIE 3.0 User's Manual, University of Maryland, Physics Department Report, 1999.

[36] Y. Papaphilippou (to be published).

[37] Recent experiments at the PSR indicate measured electrons is significantly less in TiN coated chambers than in stainless steel chambers; R. Macek (private communication).

[38] M. Blaskiewicz, in Proceedings of the 1999 Particle Accelerator Conference, New York (Ref. [8]), p. 1611.

[39] S. Y. Zhang, BNL/SNS Technical Note No. 33, 1997; BNL/SNS Technical Note No. 43, 1998; BNL/SNS Technical Note No. 61, 1999.

[40] S. Y. Zhang, in Proceedings of the 1999 Particle Accelerator Conference, New York (Ref. [8]), p. 3297. 